\title{
ANÁLISIS DEL USO DE LOS MEDIOS POR LAS GENERACIONES MÁS JÓVENES. EL MOVIMIENTO 15M Y EL UMBRELLA MOVEMENT
}

\section{Study of media habits among youth. The $15 \mathrm{M}$ and the Umbrella movements}

Ariadna Fernández-Planells

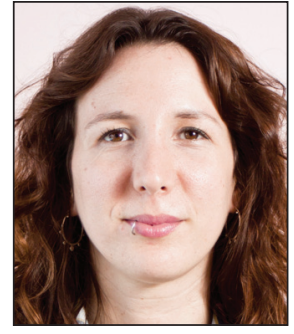

Ariadna Fernández-Planells es periodista especializada en estrategias de comunicación digital con jóvenes y movimientos sociales, investigación que combina con la docencia. Es profesora en la Universitat Pompeu Fabra donde imparte asignaturas del grado en periodismo y participa en masters y postgrados. Su principal línea de investigación se centra en la juventud y en la comunicación, especialmente en red. Forma parte del grupo de investigación Communication, Advertising and Society (CAS, UPF) y ha sido investigadora visitante en varias universidades de Perú y en la Cardiff School of Journalism, Media \& Cultural Studies en Gales. http://orcid.org/0000-0003-4600-9345

Universitat Pompeu Fabra, Departamento de Comunicación Roc Boronat, 138. 08018 Barcelona, España ariadna.fernandez@upf.edu

\section{Resumen}

El estudio de los hábitos de la población en general y de los jóvenes en particular permite anticipar el nuevo ecosistema comunicativo. Los recientes movimientos sociales en red se caracterizan por la movilización ciudadana, especialmente de los jóvenes, la ocupación del espacio público y por un uso intenso de las TIC. Es probable que a través de su observación se detecten tendencias comunicativas presentes y futuras. Se presenta un estudio longitudinal sobre los dispositivos utilizados por los activistas para informarse del Movimiento 15M en España y del Umbrella Movement en Hong Kong, llevado a cabo mediante tres encuestas realizadas en 2011 ( $N=339), 2013$ ( $N=6.167)$ y 2014 ( $N=419)$. Los resultados muestran la emergencia de los dispositivos móviles como pantallas de consulta de información, especialmente entre los jóvenes. Ello no implica el abandono de los soportes tradicionales sino que ambos tipos de pantallas conviven y se complementan, pero tales medios tradicionales están perdiendo su rol privilegiado.

\section{Palabras clave}

Medios; Medios sociales; Pantallas; Dispositivos móviles; Tabletas; Smartphones; Fuentes de información; Movimientos sociales en red, Encuestas; Movimiento 15M; Umbrella Movement.

\begin{abstract}
The study of a population's media habits and, in particular, the youth population, can allow us to predict the new communication ecosystem. Current networked social movements are characterized by citizen mobilization (especially young people), the occupation of public space, and the use of information and communication technologies (ICT). Therefore, monitoring these movements can detect technological development and communication trends. This article presents a longitudinal study of media devices used by activists to be informed about the $15 \mathrm{M}$ Movement in Spain and the Umbrella Movement in Hong Kong. The study was carried out through three surveys conducted in 2011 ( $N=339), 2013$ ( $N=6167)$ and 2014 ( $N=419)$. The results show the emergence of mobile devices as information channels, especially among young people. However, this does not imply the abandonment of traditional media; both large and small screens coexist and complement each other. Nevertheless, traditional media are losing the privileged role that they have had.
\end{abstract}

\section{Keywords}

Media; Social media; Screens; Smartphones; Tablets; Information sources; Networked social movements; Surveys; 15 May Movement; Umbrella Movement. 
Fernández-Planells, Ariadna (2015). “Análisis del uso de los medios por las generaciones más jóvenes. Eel Movimiento $15 M$ y el Umbrella Movement". El profesional de la información, v. 24, n. 4, pp. 371-379.

http://dx.doi.org/10.3145/epi.2015.jul.03

\section{Introducción}

El escenario de la comunicación está caracterizado en la actualidad por cuatro elementos bien diferenciados: hiperconectividad, información social, deslocalización, y multipantalla, lo que implica una gran variedad en los dispositivos y en la forma de acceso a la información.

El ritmo continuado de innovaciones tecnológicas y su apropiación por parte de las personas jóvenes hace que los cambios en las rutinas mediáticas sean constantes. En este sentido, el estudio de los usos comunicativos de las franjas más jóvenes de la sociedad muestra con mucha probabilidad las tendencias de los próximos años.

Desde 2009 asistimos a una oleada de movimientos sociales en red caracterizados por la ocupación del espacio público y el uso de internet como canal informativo y de movilización

En el último lustro el uso de internet y de la telefonía móvil ha experimentado un crecimiento sin precedentes (AIMC, 2013) y en paralelo ha aumentado el interés por estudiar y conocer las pantallas que la población utiliza para consumir contenidos, tanto lúdicos como informativos. La llegada a la edad adulta de la así llamada net generation (Tapscott, 1998) ha puesto de manifiesto lo que algunos analistas denominan la 'guerra de las pantallas' (Tubella; Tabernero; Dwyer, 2008), para hacer referencia a uno de los rasgos que hemos señalado antes: la multipantalla, es decir, la concurrencia de distintos dispositivos en el consumo de contenidos. En este escenario numerosos estudios (DíazNoci, 2009; Rodríguez-Martínez; Codina; Pedraza-Jiménez, 2010; Purcell et al., 2011) han detectado la creciente importancia de los dispositivos móviles como pantallas de acceso a la información, a través de portales de noticias, de medios sociales, email o de otras fórmulas.

En España el teléfono inteligente se convertía ya en 2011 en el segundo dispositivo de acceso a internet $(I A B, 2011)$, y dos años más tarde un $21 \%$ de los españoles accedía a la Red utilizando un teléfono móvil (IAB, 2013). Entre los condicionantes en la elección de la pantalla de consulta de información, la edad se revela como un factor determinante. De este modo los smartphones son el principal dispositivo para seguir la actualidad entre los jóvenes de 18 a 24 años (Newman; Levy, 2014). Según aumenta la edad, la pantalla del ordenador adquiere mayor protagonismo.

Por otra parte y paralelamente a las tendencias mediáticas descritas, desde 2009 se asiste a una nueva oleada de movimientos sociales en red (Castells, 2012), como el 15M en España, Occupy en Estados Unidos, la Primavera Árabe en Oriente Medio y Norte de África, \#YoSoy132 en México, las protestas de Brasil o, más recientemente, el Umbrella Movemement [Movimiento de los paraguas] en Hong Kong, China. A pesar de aparecer en contextos políticos y sociales muy distintos, estos movimientos comparten algunos rasgos: se han caracterizado por la movilización de los ciudadanos, especialmente jóvenes, a través de la ocupación del espacio público y por el uso de internet como canal de información y movilización.

Diversos estudios han tratado el papel de los medios sociales en estos movimientos (Fernández-Planells; FiguerasMaz; Feixa-Pàmpols, 2014; Mattoni; Treré, 2014; Bailly, 2012; Lotan et al., 2011; Costanza-Chock, 2012). Por otra parte, entre las primeras revueltas de las primaveras árabes -que algunos denominaron enfáticamente como la Twitter Revolution (Hounshell, 2011; Jurgenson, 2012)-y las movilizaciones de Hong Kong han pasado cinco años.

Este artículo realiza un estudio longitudinal para detectar la evolución en el uso de dispositivos y pantallas de consulta de información en los movimientos sociales en Red. La investigación comienza en 2011 con el Movimiento 15M en España y finaliza en 2014 con la eclosión del Umbrella Movement en Hong Kong.

Gracias a los medios sociales digitales el $15 \mathrm{M}$ creó sus propios canales de información como alternativa a los medios tradicionales

\section{El Movimiento 15M en España}

También conocido como \#spanishrevolution o Movimiento de indignados, comenzó con las masivas manifestaciones que tuvieron lugar en las principales ciudades españolas el 15 de mayo de 2011, días antes de unas elecciones regionales. En Madrid, tras la manifestación, alrededor de un centenar de jóvenes decidieron acampar en la Puerta del Sol. Un día más tarde, la Plaza de Catalunya en Barcelona se convirtió en la \#acampadabcn. En los días posteriores, las acampadas se multiplicaron por todo el país. Una gran parte de los activistas implicados eran jóvenes (Calvo-Borobia; Gómez-Pastrana; Jiménez-Sánchez, 2011; Likki, 2012). Tanto en Madrid (la misma noche en que se acampó) como en Barcelona (el 27 de mayo de 2011) hubo sendos intentos de desalojo que provocaron un "efecto llamada" entre los activistas. Finalmente, el 30 de junio de 2011 todas las acampadas habían desaparecido. Tras mes y medio de ocupaciones, entre 6 y 8,5 millones de ciudadanos españoles aseguraban haber participado de alguna manera en el 15M (Ipsos Public Affairs, 2011). 
Las aplicaciones digitales permitieron a los manifestantes difundir su propia información, optimizar su organización interna y establecer una interacción entre la acción online y offline (Micó-Sanz; CaseroRipollés, 2013). Gracias a las herramientas sociales online (Twitter, Facebook, blogs, etherpads, etc.), el Movimiento $15 \mathrm{M}$ creó sus propios canales de información como alternativa a los medios tradicionales (Fernández-Planells; Figueras-Maz; Feixa-Pàmpols, 2014).

En España existe un antecedente sobre el uso de la telefonía móvil para una movilización social. En 2004, tras los atentados del terrorismo islamista en Madrid, el gobierno afirmó que se trataba de un atentado del grupo terrorista vasco ETA. Los españoles utilizaron los móviles como canales de información alternativa (Salido-Andrés, 2006) y a través de los sms (y el "pásalo") se convocaron masivas manifestaciones. Desde entonces el teléfono móvil no había vuelto a tener tanto protagonismo como dispositivo informativo. A raíz del $15 \mathrm{M}$ aparece People witness, una red de ciudadanos con teléfonos $3 \mathrm{G}$ que retransmiten en directo manifestaciones, asambleas o acciones.

https://peoplewitness.net

\section{El Umbrella Movement en Hong Kong}

Las ocupaciones del Umbrella Movement, también conocido como Occupy Hong Kong y Occupy Central, comenzaron el 28 de septiembre de 2014 en las principales calles de los barrios de Admiralty, Mong Kok y Causeway Bay de Hong Kong. La ocupación estaba prevista para el 1 de octubre pero se avanzó tras la detención de varios estudiantes que intentaban llegar a las dependencias del legislativo autónomo. En las protestas participó el movimiento Occupy Central

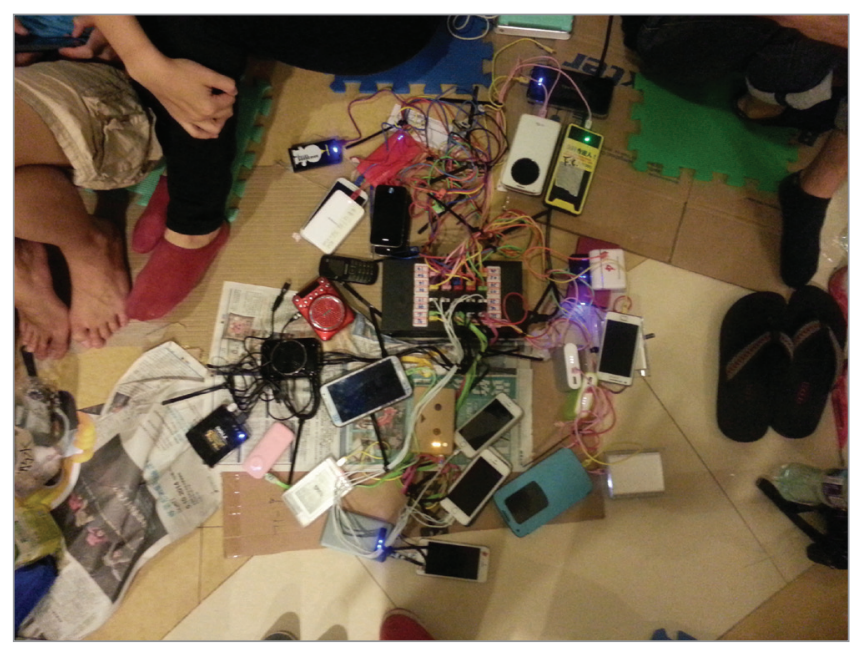

Figura 2. Punto de carga de dispositivos móviles en Admiralty, Hong Kong Fuente: Violeta Camarasa with Love and Peace, del que formaban parte la Federación de Estudiantes Universitarios y la asociación de alumnos de secundaria Scholarism. Su objetivo era conseguir retirar la reforma electoral del Congreso Nacional del Pueblo y mayor libertad en la elección de los candidatos. La polémica reforma preveía el sufragio universal en Hong Kong desde 2017 pero definiendo que los candidatos tengan que contar con la aprobación de un comité de 1.200 notables, sin posibilidad de que los ciudadanos puedan proponer nombres directamente.

Como ya pasó con el $15 \mathrm{M}$, la noche del domingo 28 de septiembre, la policía intentó desalojar a los manifestantes. Al día siguiente, miles de personas se sumaron a las ocupaciones que se alargaron hasta el 3 de diciembre, fecha en que los principales líderes se entregaron a la policía.

A pesar de tratarse de un contexto totalmente distinto, este movimiento también se caracterizó por la ocupación del espacio público y el uso de internet y los medios sociales como canales de información y movilización ciudadana. Aunque todavía no existe ningún estudio con base científica al respecto, un mero seguimiento del volumen de uso de los hashtags utilizados por el Movimiento de los paraguas y una lectura de las crónicas periodísticas de esos días, dejan patente la importancia de internet para informarse y participar en las ocupaciones de Hong Kong. Por ejemplo en Admiralty se proyectaron mensajes de apoyo enviados desde todo el mundo (figura 1). Por su parte los teléfonos se convirtieron en testigos y transmisores de lo que sucedía, llegándose a habilitar puntos de carga de estos dispositivos (figura 2).

\section{Objetivos}

Este artículo aporta datos sobre los dispositivos y pantallas asociadas utilizados por los activistas para consultar información sobre dos movimientos sociales en red surgidos con cuatro años de diferencia: el Movimiento 15M en España en 
2011 y el Umbrella Movement en Hong Kong en 2014.

Varias investigaciones han abordado el estudio de los medios utilizados por el $15 \mathrm{M}$ para difundir información (Fernández-Planells; Figueras-Maz; Feixa-Pàmpols, 2014; Micó-Sanz; Casero-Ripollés, 2013). Sin embargo, pocas se centran en los dispositivos utilizados para consultar esos medios (Monterde; Postill, 2013). En el caso del Umbrella Movement, debido a su reciente aparición, todavía no se han publicado trabajos científicos al respecto.

Este trabajo plantea tres objetivos y tres hipótesis:

Objetivo 1. Analizar la evolución en el uso de dispositivos y pantallas de consumo de información mediante el estudio de los activistas de movimientos sociales en red en dos contextos diferenciados (España y Hong Kong) entre 2011 y 2014. Concretamente, se estudia el Movimiento $15 \mathrm{M}$ en 2011 y 2013 y el Umbrella Movement en 2014.

Hipótesis 1. El estudio longitudinal entre 2011 y 2014 de dos casos muy diferenciados geográfica y culturalmente puede ayudar a identificar tendencias en el uso de los dispositivos móviles como pantallas de consumo de información frente a otro tipo de dispositivos convencionales.

Objetivo 2. Detectar si las fuentes informativas consultadas por los activistas del Movimiento $15 M$ (2011 y 2013) y del Umbrella Movement (2014) varían en función de los dispositivos y pantallas empleados.

Hipótesis 2. Debido a las especificidades de los terminales, es probable que el porcentaje de activistas que consulta los medios sociales como fuente de información sea mayor entre los usuarios de smartphones que entre los usuarios del ordenador de sobremesa y de la televisión.

Objetivo 3. Estudiar si existe relación entre la edad de los activistas, incluso en contextos geográficos y culturales diferenciados, y la elección de dispositivos y pantallas para consultar información en dos movimientos en red entre 2011 y 2014.

Hipótesis 3. Dentro de un contexto de concurrencia de pantallas, es plausible que entre los jóvenes activistas pueda confirmarse (o desmentirse) si la tendencia al uso de dispositivos móviles aumenta y por tanto disminuye el uso del ordenador de sobremesa y el portátil.

\section{Metodología}

Se utiliza la metodología cuantitativa para la obtención de resultados. Se han realizado tres encuestas para conocer el perfil de edad de los activistas encuestados y el uso de dispositivos y pantallas para consultar información. Esta técnica se ha complementado con la observación participante y entrevistas en profundidad que han permitido matizar y comprender algunos de los resultados obtenidos a través de las encuestas.

\section{Encuesta 1}

Coincidiendo con las acampadas del $15 \mathrm{M}$ en España, se realizó una primera encuesta entre el 28 de mayo y el 25 de junio de 2011 en la Plaza de Catalunya de Barcelona. El 90\% de los cuestionarios se completaron en papel en la misma acampada, aunque algunos también fueron contestados por correo electrónico. El tiempo promedio de realización de la encuesta fue de aproximadamente 10 minutos. En total se realizaron 420 encuestas de las que finalmente se validaron 339 ( $\mathrm{N}=339$ ). Para validar los cuestionarios, el encuestado tenía que haber respondido a todas las preguntas y las respuestas tenían que ser coherentes entre sí.

La encuesta se diseñó ad hoc para este estudio. Con el objetivo de validarla, se realizó un pre-test para identificar posibles anomalías. Tras realizar algunas modificaciones, se distribuyó la versión final. Para analizar el uso de las distintas pantallas, los encuestados debían escoger entre un listado que incluyó la pantalla del ordenador de sobremesa, la del portátil o notebook, la del teléfono móvil o smartphone y la de la tableta.

Los participantes fueron seleccionados mediante un muestreo incidental o de conveniencia. No existían datos sobre el número de personas que asistieron a las plazas cada día ni sobre el perfil socio demográfico de los participantes en el $15 \mathrm{M}$. Por ello, en la selección de la muestra, se trató de ser lo más riguroso posible: se dividió la plaza en cuatro zonas, que correspondían a las áreas alrededor de las cuatro entradas principales. De este modo se cubrían todas las comisiones instaladas en ella. Se trató de encuestar a los participantes de todas las zonas y el único criterio de selección fue que estuviesen en la plaza participando como visitantes, miembros de una comisión o acampados. Teniendo en cuenta que el universo no puede definirse correctamente, cuando este artículo se refiere a activistas del $15 \mathrm{M}$, sólo se hace referencia al universo de la muestra, es decir, a aquellos activistas encuestados. Éstos eran principalmente jóvenes de entre 18 y 30 años (figura 3). 


\section{Encuesta 2}

Se realizó una segunda encuesta coincidiendo con el segundo aniversario del Movimiento $15 \mathrm{M}$ en mayo de 2013. Se mantuvieron las preguntas realizadas en la encuesta de 2011. Sin embargo, debido a la idiosincrasia del Movimiento durante el segundo aniversario, y a la desaparición de las ocupaciones, se creó un cuestionario online y se difundió entre el 14 y el 30 de mayo de 2013 a través de medios sociales y Whatsapp. Pronto saltó a Menéame y algunos medios como Eldiario.es, El país o la cadena SER dieron difusión a la encuesta. En tan sólo 24 horas se recibieron más de 4.000 respuestas. Finalmente se recogieron 6.167 ( $\mathrm{N}=6.167)$. Debido al método de difusión escogido, se trató de un muestreo por bola de nieve. El 0,4\% de los participantes era menor de 18 años, el $32,9 \%$ entre 18 y 30 , el $36,6 \%$ entre 31 y 40 y el $30,1 \%$ de más de 41 años (figura 3).

\section{En 2011 los activistas del 15M utilizaron para informarse principalmente el orde- nador portátil, seguido del de sobreme- sa, la televisión y el teléfono móvil}

\section{Encuesta 3}

Se realizó una tercera encuesta coincidiendo con el surgimiento del Umbrella Movement en Hong Kong. Se aplicó el mismo cuestionario traducido al inglés y al chino. Se creó online y se difundió en los medios sociales. Además se colgaron carteles en las acampadas de Hong Kong con un código QR que dirigía a la encuesta. Entre el 30 de septiembre y el 23 de noviembre de 2014 se recogieron 419 encuestas ( $\mathrm{N}=419)$. Como en la segunda encuesta, se trató de un muestreo por bola de nieve. El 5,7\% de los participantes era menor de 18 años, el 41,9\% entre 18 y 30 , el 27,4\% entre 31 y 40 y el $24,7 \%$ de más de 41 años (figura 3 ).

\section{Los dispositivos móviles como pantallas de consulta de información}

Los datos de esta investigación reflejan la aparición y consolidación de los dispositivos móviles como pantallas de acceso a la información entre 2011 y 2014. En 2011 los activistas del $15 \mathrm{M}$ en Barcelona utilizaron principalmente la pantalla del ordenador portátil para informarse del Movimiento, seguido del ordenador de sobremesa, la televisión y el teléfo- no móvil. El uso de tabletas fue meramente testimonial con sólo un 2,6\% de usuarios (figura 4). El elevado porcentaje de consulta de información a través de la televisión pudo deberse a la cobertura informativa realizada. Los entrevistados destacaron la retransmisión de la cadena de televisión de ámbito nacional Antena 3 y de la regional TV3 sobre el intento de desalojo de la Plaza de Catalunya. Además, debido a la ocupación del espacio público, las acampadas protagonizaron telediarios y tertulias.

En el segundo aniversario del $15 \mathrm{M}$, la cobertura mediática fue menos intensa, lo que explicaría el descenso en los porcentajes de consulta de información a través de la pantalla de televisión y, en contraposición, el aumento de los porcentajes de los dispositivos móviles. Tal y como puede verse en la figura 4, el uso del teléfono móvil como pantalla de consulta de información sube a costa de la televisión, del ordenador de sobremesa y del portátil.

\section{En 2014 el smartphone es el principal dispositivo de consulta de información sobre el Umbrella Movement, le siguen ordenador portátil y tableta, y atrás quedan televisión y ordenador de so- bremesa}

En 2014, en un contexto distinto pero también dentro de los llamados movimientos en red, esta tendencia se consolida, hasta el punto de que el teléfono inteligente se convierte en el primer dispositivo y pantalla para consultar información sobre el Umbrella Movement. Le siguen de cerca la pantalla del ordenador portátil y de la tableta pero atrás quedan la televisión o el ordenador de sobremesa (figura 4).

Los resultados expuestos hasta este punto plantean un nuevo interrogante: ¿varían las fuentes informativas consultadas por los activistas para informase de estos movimientos 
Tabla 1. Uso de fuentes de información según los dispositivos utilizados para informarse del Movimiento 15 M (2011 y 2013) y del Umbrella Movement (2014).

\begin{tabular}{|c|c|c|c|c|c|c|c|c|c|c|c|c|c|c|c|c|c|c|c|}
\hline \multirow{3}{*}{\multicolumn{2}{|c|}{$\begin{array}{c}\text { Pantallas de informa- } \\
\text { ción }\end{array}$}} & \multicolumn{18}{|c|}{ Fuentes de información } \\
\hline & & \multicolumn{3}{|c|}{ Twitter } & \multicolumn{3}{|c|}{ Facebook } & \multicolumn{3}{|c|}{ Medios online } & \multicolumn{3}{|c|}{ Prensa } & \multicolumn{3}{|c|}{ Radio } & \multicolumn{3}{|c|}{ Televisión } \\
\hline & & 2011 & 2013 & 2014 & 2011 & 2013 & 2014 & 2011 & 2013 & 2014 & 2011 & 2013 & 2014 & 2011 & 2013 & 2014 & 2011 & 2013 & 2014 \\
\hline \multirow{2}{*}{ Televisión } & $\mathrm{N}$ & 10 & 783 & 58 & 59 & 998 & 117 & 67 & 1.319 & 96 & 27 & 691 & 59 & 16 & 732 & 38 & 53 & 1.193 & 93 \\
\hline & $\%$ & 11,2 & 54 & 46,8 & 66,3 & 68,9 & 94,4 & 75,3 & 91 & 77,4 & 30,3 & 47,7 & 47,6 & 18,0 & 50,5 & 30,6 & 59,6 & 82,3 & 75 \\
\hline \multirow{2}{*}{$\begin{array}{l}\text { Ordenador } \\
\text { sobremesa }\end{array}$} & $\mathrm{N}$ & 31 & 1.997 & 100 & 91 & 2.419 & 190 & 125 & 3.151 & 145 & 24 & 1.006 & 72 & 19 & 966 & 48 & 52 & 1.612 & 100 \\
\hline & $\%$ & 21,2 & 57,5 & 48,8 & 62,3 & 69,6 & 92,7 & 85,6 & 90,7 & 70,7 & 16,4 & 28,9 & 35,1 & 13,0 & 27,8 & 23,4 & 35,6 & 46,4 & 48,8 \\
\hline \multirow{2}{*}{$\begin{array}{l}\text { Portátil o note- } \\
\text { book }\end{array}$} & $\mathrm{N}$ & 46 & 2.205 & 141 & 136 & 2.564 & 226 & 174 & 3.168 & 181 & 40 & 1.015 & 83 & 18 & 992 & 57 & 51 & 1.601 & 117 \\
\hline & $\%$ & 23 & 62,6 & 57,6 & 68 & 72,8 & 92,2 & 87 & 89,9 & 73,9 & 20 & 28,8 & 33,9 & 9,0 & 28,2 & 23,3 & 25,5 & 45,4 & 47,8 \\
\hline \multirow{2}{*}{ Tablet } & $\mathrm{N}$ & 5 & 535 & 80 & 6 & 478 & 120 & 11 & 709 & 92 & 2 & 259 & 52 & 0 & 231 & 27 & 4 & 375 & 64 \\
\hline & $\%$ & 38,5 & 70,3 & 60,2 & 46,2 & 62,8 & 90,2 & 84,6 & 93,2 & 69,2 & 15,4 & 34 & 39,1 & 0 & 30,4 & 20,3 & 30,8 & 49,3 & 48,1 \\
\hline \multirow{2}{*}{ Smartphone } & $\mathrm{N}$ & 19 & 2.128 & 181 & 31 & 2.029 & 341 & 38 & 2.545 & 251 & 6 & 852 & 120 & 2 & 790 & 80 & 8 & 1.290 & 178 \\
\hline & $\%$ & 45,2 & 75,5 & 48,9 & 73,8 & 72 & 92,2 & 90,5 & 90,3 & 67,8 & 14,3 & 30,2 & 32,4 & 4,8 & 28 & 21,6 & 19 & 45,8 & 48,1 \\
\hline
\end{tabular}

en función del dispositivo empleado? Los datos muestran que en 2011 el porcentaje de usuarios que consultan Facebook, Twitter y medios digitales como fuentes de información es mayor entre los usuarios de smartphones (tabla 1). El portátil también es uno de los principales dispositivos de consulta de estos medios. En contraposición, entre los usuarios de la televisión como pantalla informativa encontramos a la audiencia principal de los medios tradicionales (televisión, prensa en papel y radio). En 2013, se mantienen estas tendencias pero destaca el crecimiento del número de usuarios que consultan Twitter, Facebook y los medios digitales a través de tabletas.

\section{El uso del ordenador de sobremesa en} 2014 aumenta según aumenta la edad de los encuestados

En el caso de Hong Kong y los activistas del Umbrella Movement, son los usuarios de televisión la audiencia principal de todas las fuentes informativas excepto de Twitter. Entre los que utilizan la tableta encontramos el mayor porcentaje de usuarios de esta red de microblogging (tabla 1).

\section{Edad en la elección del dispositivo}

Mientras que en 2011 la edad no aparece como un factor significativo en el uso de los dispositivos y pantallas, en 2013 sucede todo lo contrario: la asociación entre edad y uso de pantallas es altamente significativa en todos los casos, tanto en la tableta $\left(\mathrm{Chi}^{2}=56,019, \mathrm{p}<0,001\right)$, como el teléfono móvil $\left(\mathrm{Chi}^{2}=120,522, \mathrm{p}<0,001\right)$, el ordenador portátil $\left(\mathrm{Chi}^{2}=193,543, \mathrm{p}<0,001\right)$, el ordenador de sobremesa $\left(\mathrm{Chi}^{2}=86,174, \mathrm{p}<0,001\right)$ o la televisión $\left(\mathrm{Chi}^{2}=43,010\right.$, $\mathrm{p}<0,001)$.

Tal significación indica que entre los activistas de entre 31 y 40 años, el porcentaje de uso de la tableta como pantalla de consulta de información sobre el $15 \mathrm{M}$ es mayor que entre los que están por debajo o por encima de esa franja de edad. Lo mismo sucede con el teléfono móvil, aunque en este caso la distancia con los activistas de entre 19 y 30 años se acorta y en cambio aumenta con los de más de 41 años. En el caso del ordenador portátil, son los que tienen entre 19 y 30 años los que más utilizan estos dispositivos en comparación con el resto de encuestados. Y la elección del ordenador de sobremesa y de la televisión como pantallas de consulta de información aumenta según aumenta la edad (tabla 2).

\section{El teléfono móvil pasa de ser la cuarta pantalla para consultar información en 2011 a ser la primera en 2014}

Los datos de 2014 en un nuevo contexto muestran que la relación entre el uso de dispositivos para consulta de información y la edad es significativa en el caso del ordenador portátil $\left(\mathrm{Chi}^{2}=10,874, \mathrm{p}<0,012\right)$ y del ordenador de sobremesa $\left(\mathrm{Chi}^{2}=11,223, \mathrm{p}<0,011\right)$ en el mismo sentido que en 2013. Es decir, el porcentaje más elevado de activistas que utilizan el portátil es en edades entre 19 y 30 años, seguido de los menores de 18 , los mayores de 41 y por último de entre 31 y 40 años. Por el contrario, el uso del ordenador de sobremesa -como en 2013- aumenta según aumenta la edad de los encuestados. Aunque los resultados no sean significativos, destaca el uso del teléfono móvil entre los menores de 30 años, mientras que la tableta es el dispositivo más utilizado entre los mayores de 41 años y la televisión entre los menores de 18 y los mayores de 41 (tabla 2).

\section{Discusión y conclusiones}

\section{Emergencia y consolidación}

Los resultados muestran la emergencia y consolidación de los móviles como pantallas de acceso a la información. El teléfono móvil pasa de ser el cuarto dispositivo para con- 
Tabla 2. Uso de pantallas para consultar información según la edad de los activistas encuestados en el Movimiento $15 M$ (2011 y 2013 ) y el Umbrella Movement (2014).

\begin{tabular}{|c|c|c|c|c|c|c|c|c|c|c|c|c|c|c|c|c|}
\hline \multirow{2}{*}{\multicolumn{2}{|c|}{ Edad }} & \multicolumn{3}{|c|}{ Tableta } & \multicolumn{3}{|c|}{$\begin{array}{l}\text { Teléfono móvil o } \\
\text { smartphone }\end{array}$} & \multicolumn{3}{|c|}{ Portátil o notebook } & \multicolumn{3}{|c|}{$\begin{array}{l}\text { Ordenador de sobre- } \\
\text { mesa }\end{array}$} & \multicolumn{3}{|c|}{ TV } \\
\hline & & 2011 & 2013 & 2014 & 2011 & 2013 & 2014 & 2011 & 2013 & 2014 & 2011 & 2013 & 2014 & 2011 & 2013 & 2014 \\
\hline \multirow{2}{*}{$<18$} & $\mathrm{~N}$ & 1 & 1 & 5 & 1 & 11 & 25 & 8 & 15 & 17 & 2 & 11 & 10 & 3 & 5 & 9 \\
\hline & $\%$ & 10 & 4,3 & 18,5 & 10 & 47,8 & 92,6 & 80 & 65,2 & 63 & 20 & 47,8 & 37 & 30 & 21,7 & 33,3 \\
\hline \multirow{2}{*}{$\begin{array}{l}19- \\
30\end{array}$} & $\mathrm{~N}$ & 9 & 151 & 51 & 36 & 932 & 165 & 171 & 1.303 & 119 & 126 & 933 & 76 & 78 & 390 & 51 \\
\hline & $\%$ & 3,1 & 8,1 & 29,3 & 12,4 & 49,8 & 94,8 & 59 & 69,6 & 68,4 & 43,4 & 49,8 & 43,7 & 26,9 & 20,8 & 29,3 \\
\hline \multirow{2}{*}{$\begin{array}{l}31- \\
40\end{array}$} & $\mathrm{~N}$ & 3 & 319 & 41 & 5 & 1.139 & 104 & 22 & 1236 & 59 & 17 & 1.274 & 58 & 7 & 471 & 33 \\
\hline & $\%$ & 8,1 & 15,3 & 35,7 & 13,5 & 54,8 & 90,4 & 59,5 & 59,5 & 51,3 & 45,9 & 61,3 & 50,4 & 18,9 & 22,7 & 28,7 \\
\hline \multirow{2}{*}{$>41$} & $\mathrm{~N}$ & 0 & 248 & 38 & 0 & 637 & 87 & 0 & 800 & 54 & 1 & 1.094 & 64 & 1 & 509 & 31 \\
\hline & $\%$ & 0 & 14,5 & 37,3 & 0 & 37,2 & 85,3 & 0 & 46,7 & 52,9 & 50 & 63,9 & 62,7 & 50 & 29,7 & 30,4 \\
\hline
\end{tabular}

sultar información en 2011 a ser el primero en 2014, y la tableta pasa de un uso del $2,6 \%$ al $11,6 \%$, Ilegando a utilizarse más que la televisión. Por el contrario decrece el uso de la televisión, el ordenador de sobremesa y especialmente el portátil.

El estudio del Movimiento $15 \mathrm{M}$ y del Umbrella Movement muestra cómo los teléfonos móviles recuperan su faceta informativa y movilizadora gracias al acceso a internet, a los medios sociales y a las aplicaciones de mensajería instantánea como Whatsapp o WeChat. Entre los jóvenes de estos movimientos, los dispositivos móviles se han convertido en herramientas de acceso a la información y en testigos y difusores de noticias.

Los dispositivos móviles se han convertido en herramientas de acceso a la información y en testigos y difusores de noticias

\section{Factor edad en la elección de la pantalla}

Confirmando anteriores estudios, la edad aparece como factor significativo en la elección de la pantalla de consulta de información, sin embargo no en todos los casos ni siempre de la manera esperada (tabla 2).

Los resultados de 2013 muestran que aunque los jóvenes activistas de entre 19 y 30 años utilizan ampliamente los dispositivos móviles para obtener información del 15M, son los activistas de entre 31 y 40 años los principales usuarios. Los datos de 2014 confirmarían la hipótesis inicial de este estudio: los jóvenes de entre 19 y 30 años son los que más escogen el smartphone como pantalla de acceso a la información. El uso de la tableta en cambio está más extendido entre los mayores de 31 años.

\section{Medios y dispositivos}

Las fuentes consultadas por los activistas para informarse de los movimientos en red varían en función del dispositivo. Esto implica que los usuarios de dispositivos móviles son los principales usuarios de medios sociales y digitales, espe- cialmente de Twitter. En cambio los usuarios de dispositivos y pantallas convencionales son los principales usuarios de medios tradicionales (televisión, prensa en papel y radio).

\section{Pérdida del rol privilegiado de los medios tradiciona- les}

Los resultados demuestran que los dispositivos móviles se han convertido en las principales pantallas de acceso a la información entre los activistas de los movimientos sociales en red analizados. Sin embargo esto no implica el abandono de los soportes tradicionales. Parece que ambos tipos de pantallas conviven y se complementan. Lo que sucede es que los medios tradicionales han perdido, tal vez de forma irreversible, el rol privilegiado del que disponían hasta ahora.

Las fuentes consultadas por los activistas para informarse varían en función del dispositivo

En efecto, el $13,4 \%$ en 2011 , el $49,4 \%$ en 2013 y el $86,6 \%$ en 2014 utiliza al menos un dispositivo móvil y un dispositivo convencional para informarse. Y desde 2011 hasta 2014 la mayoría de activistas encuestados utilizaron al menos dos o más dispositivos para consultar información (tabla 3). Por lo

Tabla 3. Pantallas utilizadas para consultar información en 2011, 2013 y 2014

\begin{tabular}{|l|c|c|c|}
\hline No de pantallas & $\mathbf{2 0 1 1}(\%)$ & $\mathbf{2 0 1 3}(\%)$ & $\mathbf{2 0 1 4}(\%)$ \\
\hline No usa & 0,9 & 3,8 & 2,2 \\
\hline Usa 1 & 60,8 & 34,4 & 13,6 \\
\hline Usa 2 & 31,0 & 34,9 & 30,1 \\
\hline Usa 3 & 6,2 & 17,6 & 28,7 \\
\hline Usa 4 & 1,2 & 7,1 & 15,6 \\
\hline Usa 5 & 0,0 & 2,1 & 7,4 \\
\hline Usa 6 & 0,0 & 0,0 & 2,4 \\
\hline Total & 100 & 100 & 100 \\
\hline
\end{tabular}


tanto estos datos apuntarían al uso y coexistencia de múltiples pantallas, bien de manera simultánea (multitasking), bien en momentos distintos.

\section{Validez y extrapolaciones plausibles}

Los resultados de este artículo son relevantes para la investigación sobre el uso de dispositivos de consulta de información entre los jóvenes. En concreto, entre los activistas de los recientes movimientos en red. Estos movimientos han centrado la atención de la academia por, entre otras cosas, las innovaciones introducidas en el ámbito comunicativo. Es tan importante conocer qué medios escogen los jóvenes activistas para mantenerse informados como conocer a través de qué dispositivos consultan dichos medios. Y hasta el momento existen muy pocas investigaciones que aborden el estudio desde este prisma.

La emergencia de los dispositivos móviles no implica el abandono de los soportes tradicionales sino la coexistencia de múltiples pantallas, de manera simultánea o en momentos distintos

Evidentemente este análisis presenta limitaciones. El hecho de que la investigación se centre en los movimientos sociales en red implica el estudio de dos sociedades muy distintas. A pesar de ello resulta de especial interés conocer los cambios que se producen en la elección de dispositivos y pantallas desde una perspectiva temporal (2011-2014) y social (España - Hong Kong). Por otro lado la selección de la muestra, debido a la dificultad para identificar previamente a los activistas, también conlleva limitaciones. Sin embargo tanto su amplitud como su singularidad y temporalidad otorgan relevancia a los resultados.

\section{Futuras investigaciones}

Matizar los resultados mediante la combinación de métodos cuantitativos y cualitativos permitiría detectar los usos específicos de cada dispositivo. Es decir, estudiar qué funciones y valores se atribuyen a cada pantalla, en qué situaciones se utiliza una u otra y qué tipo de contenidos se consultan. Asimismo, realizar una encuesta global en que participen activistas de todos los movimientos sociales en red, ofrecería una radiografía actualizada de las tendencias comunicativas entre los jóvenes que protagonizan los movimientos sociales actuales.

\section{Nota}

Este trabajo forma parte del proyecto Audiencias activas y periodismo. Interactividad, integración en la web y buscabilidad de la información periodística. CSO2012-39518-C04-02. Plan Nacional de I+D+i, Ministerio de Economía y Competitividad (España).

\section{Bibliografía}

Asociación para la Investigación de Medios de Comunicación (AIMC) (2013). Resumen general de resultados EGM. Octubre de 2012 a mayo de 2013.
http://www.aimc.es/Entrega-de-resultados-EGM-2\%C2\%AAola,1287.html

Bailly, Jordan (2012). The impact of social media on social movements: A case study of the 2009 Iranian green movement and the 2011 Egyptian revolution. Doctoral thesis. Washington State University, Department of Political Science. https://goo.gl/rDfidw

Calvo-Borobia, Kerman; Gómez-Pastrana, Teresa; JiménezSánchez, Luis-Mena (2011). Movimiento 15M: ¿quiénes son y qué reivindican?. Fundación Alternativas.

http://www.fundacionalternativas.org/laboratorio/librose-informes/libros/especial-15-m

Castells, Manuel (2012). Redes de indignación y esperanza. Madrid: Alianza Editorial. ISBN: 9788420609607

Costanza-Chock, Sasha (2012). "Mic Check! Media cultures and the occupy movement". Social movement studies, v. 11, n. 3-4, pp. 375-385.

http://web.mit.edu/schock/www/docs/pubs/mic-check2012-costanza-chock.pdf

http://dx.doi.org/10.1080/14742837.2012.710746

Díaz-Noci, Javier (2009). “2018: ¿Diarios en dispositivos móviles? Libro electrónico, tinta electrónica y convergencia de la prensa impresa y digital". El profesional de la información, v. 18, n. 3, pp. 301-307.

http://www.elprofesionaldelainformacion.com/contenidos/2009/ mayo/07.html

http://dx.doi.org/10.3145/epi.2009.may.07

Fernández-Planells, Ariadna; Figueras-Maz Mònica; FeixaPàmpols, Carles (2014). "Communication among young people in the \#spanishrevolution. Uses of online-offline tools to obtain information about the \#acampadabcn". New media \& society, v. 16, n. 8.

http://dx.doi.org/10.1177/1461444814530097

Hounshell, Blake (2011). "The revolution will be twitted". Saudiwave.

http://www.saudiwave.com/pdf/Press/the-revolution-willbe-tweeted.pdf

IAB Spain Research (2011). III Estudio sobre redes sociales en internet. IAB Spain Research; Elogia Marketing Singular. http://www.iabspain.net/wp-content/uploads/downloads/2012/04/ III-Estudio-sobre-redes-sociales-en-Internet.pdf

IAB Spain Research (2013). IV Estudio anual redes sociales. http://www.iabspain.net/redes-sociales

Ipsos Public Affairs (2011). Cantidad y calidad de apoyos al Movimiento 15M. Madrid: Ipsos.

Jurgenson, Nathan (2012). "Twitter revolution". En: Ritzer, George (ed.). The Wiley-Blackwell encyclopedia of globalization. Blackwell Publishing Ltd. ISBN: 9781405188241 http://dx.doi.org/10.1002/9780470670590.wbeog921

Likki, Tinna (2012). 15M revisited: A diverse movement united for change. Fundación Alternativas.

http://www.falternativas.org/laboratorio/libros-e-informes/ zoom-politico/15m-revisited-a-diverse-movement-united-forchange 
Lotan, Gilad; Graeff, Erhardt; Ananny, Mike; Gaffney, Devin; Pearce, Ian; Boyd, Danah (2011). "The revolutions were tweeted: Information flows during the 2011 Tunisian and Egyptian revolutions web ecology project". International journal of communication, v. 5, pp. 1375-1406.

http://ijoc.org/index.php/ijoc/article/view/1246/643

Mattoni, Alice; Treré, Emiliano (2014). “Media practices, mediation processes, and mediatization in the study of social movements". Communication theory, v. 24, n. 3, pp. 252-271. http://dx.doi.org/10.1111/comt.12038

Micó-Sanz, Josep-Lluís; Casero-Ripollés, Andreu (2012). "Political activism online: organization and media relations in the case of 15M in Spain". Information, communication \& society, v. 17, n. 7, pp. 858-871.

http://dx.doi.org/10.1080/1369118X.2013.830634

Monterde, Arnau; Postill, John (2013). “Mobile ensembles: The uses of mobile phones for social protest by Spain's indignados". En: Goggin, Gerard; Hjorth, Larissa (eds.). Routledge companion to mobile media. ISBN: 9780415809474 http://openaccess.uoc.edu/webapps/o2/bitstream/10609/39161/1/ Monterde_TheRoutledge2014_Mobile.pdf

Newman, Nic; Levy, David A. (eds.) (2014). Reuters Institute digital news report 2014. Tracking the future of news. Reuters Institute.
https://goo.gl/wceBV8

Purcell, Kristen; Rainie, Lee; Mitchell, Amy; Rosenstiel, Tom; Olmstead, Kenny (2010). Understanding the participatory news consumer. How internet and cell phone users have turned news into a social experience. Pew Research Center. http://goo.gl/a2S2Y9

Rodríguez-Martínez, Ruth; Codina Lluís; Pedraza-Jiménez, Rafael (2010). "Cibermedios y web 2.0: modelo de análisis y resultados de aplicación". El profesional de la información, v. 19, n. 1 , pp. $35-44$.

http://www.elprofesionaldelainformacion.com/contenidos/2010/ enero/05.pdf

http://dx.doi.org/10.3145/epi.2010.ene.05

Salido-Andrés, Noelia (2006). "Del 11M al 14M: jornadas de movilización social". En: Vara-Miguel, Alfonso (coord.). La comunicación en situaciones de crisis: del 11-M al 14-M. Actas del Congreso internacional de comunicación. Facultad de Comunicación, Universidad de Navarra, pp. 271-284. ISBN: 8431323744

Tapscott, Don (1998). Growing up digital: The rise of the net generation. New Cork: McGraw-Hill. ISBN: 0070633614

Tubella, Imma; Tabernero, Carlos; Dwyer, Vincent (2008). Internet y televisión: la guerra de las pantallas. Barcelona: Ariel. ISBN: 9788434442818

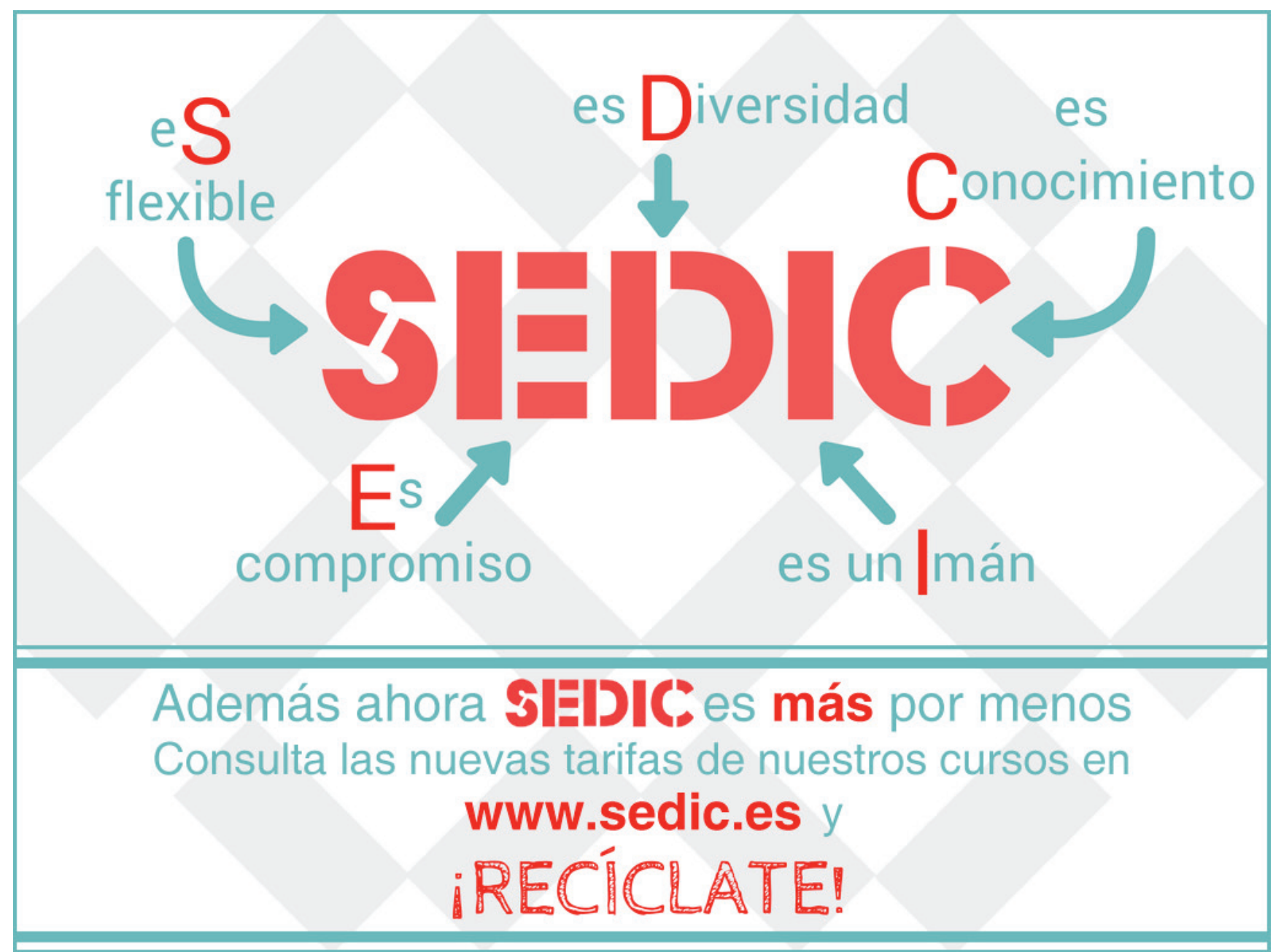

